\title{
Fibroblast growth factor 2 supports osteoblastic niche cells during hematopoietic homeostasis recovery after bone marrow suppression
}

Kyung-Ae Yoon, YeonSung Son, Young-Jin Choi, Joo-Hyun Kim and Je-Yoel Cho*

\begin{abstract}
Background: Hematopoietic stem cell (HSC) maintenance requires a specific microenvironment. HSC niches can be activated by tissue damaging chemotherapeutic drugs and various cell signaling molecules such as SDF-1 and FGF, which might also result in bone marrow stress. Recent research has insufficiently shown that endosteal osteolineage cells and other niche constituents recover after marrow injury.

Methods: We investigated the role of FGF2 in the osteoblastic niche cells during hematopoietic homeostasis recovery after bone marrow injury. Mice were treated with 5 -fluorouracil (5FU) to eliminate actively cycling cells in the bone marrow. Primary osteoblasts were isolated and subjected to cell culture. Real-time PCR, western blot and immunohistochemical staining were performed to study niche-related genes, osteoblast markers, and FGF2 signaling. Proliferation rate were analyzed by marker gene Ki67 and colony formation assay. Also, osterix-positive osteoprogenitor cells were isolated by FACS from Osx-GFP-Cre mice after 5FU treatment, and subjected to RNA-sequencing and analyzed for Fgf receptors and niche markers.

Results: The endosteal osteolineage cells isolated from 5FU-treated mice showed increased expression of the niche-related genes Sdf-1, Jagged-1, Scf, N-cad, Angpt1 and Vcam-1 and the osteoblast marker genes Osx, Opn, Runx2, and Alp, indicating that BM stress upon 5FU treatment activated the osteoblastic niche. Endosteal osteoblast expanded from a single layer to several layers 3 and 6 days after $5 F U$ treatment. During the early recovery phase in 5FU-activated osteoblastic niches increased FGF2 expression and activated its downstream pERK. FGF2 treatment resulted in increased proliferation rate and the expression of niche marker genes in 5FU-activated osteoblastic niche cells. RNA-seq analysis in Osterix-positive osteoprogenitor cells isolated from 5FU-treated Osx-GFP mice showed significantly increased expression of Fgf receptors Fgfr1, 2 and 3. Although osteoblastic niche cells were damaged by $5 F U$ treatment in the beginning, the increased number of $\mathrm{OB}$ layers in the recovery phase may be derived from resident osteoprogenitor cells by FGF2 activation under stress.
\end{abstract}

Conclusions: Taken together, FGF2 signaling can regulate osteoblastic niche cells to support HSC homeostasis in response to bone marrow damage.

Keywords: FGF2, Hematopoietic stem cell, Niche, Osteoblast, Osteoprogenitor

\footnotetext{
* Correspondence: jeycho@snu.ac.kr

Department of Biochemistry, BK21 PLUS Program for Creative Veterinary Science Research and Research Institute for Veterinary Science, College of Veterinary Medicine, Seoul National University, 1 Gwanak-ro, Gwanak-gu, Seoul, South Korea
} 


\section{Background}

Hematopoietic stem cells (HSCs) are cells capable of differentiating into all blood cell lineages, and they are tightly regulated by the local niche to maintain homeostasis. HSC niches can be compromised by several different cell types, including endosteal osteolineage cells and vascular sinusoidal endothelial cells in the bone marrow. Regulatory signal emanating from surrounding cells within the niche and physiologic cues could affect HSC quiescence, proliferation and differentiation [1, 2]. Under steady state conditions, the majority of HSCs are quiescent but can be activated to proliferate and differentiate after suppression by infection, radiation, or chemotherapeutic drug treatment, such as cyclophosphamide and 5fluorouracil (5FU) [3-6]. Chemotherapeutic drug treatment for hematological diseases induce damage to the bone marrow (BM) microenvironment through multiple BM stress responses. Under BM stress, cycling cells are eliminated and surviving quiescent long-term hematopoietic stem cells (LT-HSCs) are activated to expand via a complex mechanism [7-9].

Fibroblast growth factors (FGFs) are secreted molecules that regulate a variety of biological processes, such as cell proliferation, differentiation, and migration during both embryonic and adult development [10]. FGFs bind FGF receptors (FGFRs) and heparin sulfate proteoglycans (HSPGs) to modulate downstream biological processes. FGFRs include 4 tyrosine kinases designated FGFR1-4. Interactions of FGF and the receptor display different ligand-binding and biological functions [11]. The various subtypes of FGFRs form via splicing in immunoglobulin domain three of FGFR1, FGFR2 and FGFR3, but not FGFR4. Therefore, there are several FGFR1-3 subtypes, such as FGFR1-3 IIIb and FGFR1-3 IIIc.

Several studies have suggested that FGF signaling has important roles in HSC maintenance. FGF2 knock-out (KO) BM stromal cells weakly support HSC maintenance [12]. Under irradiation conditions, FGF2 $\mathrm{KO}$ mice showed delayed recovery of BM LSK $\left(\mathrm{Lin}^{-} \mathrm{Sca}-1^{+} \mathrm{cKit}^{+}\right)$ HSPC cells and a reduced population of CD34 ${ }^{-}$LSK cells (enriched with LT-HSCs) [13]. The microenvironment indirectly mediates FGF signaling, as HSCs expanded only in unfractionated BM culture [14]. BM suppression induced HSC activation by FGF secreted by megakaryocytes [9]. While it is known that osteoprogenitor cells have important roles in HSC maintenance, the endosteal osteoblasts (OBs) significantly expanded upon BM stress [15-17]. FGF signaling in bone is related to skeletal development and disease [18]. Deletion of the niche factor SDF-1 using osterix (Osx)-cre led to significant HSC mobilization [19]. Deletion of Osx in bone also affects hematopoiesis in the BM [20].

Here, we studied whether FGF2 could support osteoprogenitor cell-derived endosteal osteoblastic cells to recover HSPCs and modify the microenvironment to support HSPC recovery after 5FU-induced stress. The results showed that FGF2 mediates endosteal osteoblastic niche cell proliferation upon 5FU-induced stress. Activated FGF signaling increased pERK levels early after acute injury and increased Fgfr expression in the $\mathrm{OB}$ niche cells. In particular, our study suggests that, after BM stress, Osx-positive osteoprogenitor cells are activated by increasing Fgfr expression to proliferate in response to FGF2.

\section{Methods}

\section{Animals and 5FU treatment}

C57BL/6 mice were used to isolate primary osteoblasts, and the hematopoietic stem cell niche was activated by 5 fluorouracil (5FU) (Sigma-Aldrich, St. Louis, MO, USA). Mice were injected once with $5 \mathrm{FU}$ at a dose of $150 \mu \mathrm{g} / \mathrm{g}$ body weight. Bone tissues were harvested to isolate primary osteoblasts (endosteal osteolineage cells) at various time points after 5FU treatment. Osx-GFP-Cre mouse were purchased from The Jackson Laboratory.

\section{Primary osteoblast isolation}

Primary osteoblast cells were isolated as previously reported by our group [21]. Briefly, crushed bones (femora, tibiae, and humeri) were removed from control or 5FU-treated mice and washed with PBS (GIBCO, Grand Island, NY) with 2\% FBS (Hyclone, South Logan, UT) until the bone chips were white. Endosteal osteoblasts were released by enzyme digestion for 90 mins at $37{ }^{\circ} \mathrm{C}$ at $150 \mathrm{rpm}$ with $3 \mathrm{mg} / \mathrm{ml}$ type I collagenase (Worthington, Lakewood, NJ) and $0.05 \%$ dispase (GIBCO) in PBS with 20\% FBS. The released endosteal osteoblasts were washed in PBS $+2 \%$ FBS, and after removing the residual bone material osteoblasts were made into a single cell suspension by filtering through a $45-\mu \mathrm{m}$ filter (BD Bioscience, San Jose, CA). In some experiments, osteoblastic cells were also isolated by the explant culture method in a 12-well plate containing the same weight of bone tissues per well. After primary osteoblasts became confluent within 5 to 10 days in the presence of FGF2 (R\&D systems, Minneapolis, MN) or without FGF2, osteoblast colonies originating from bone fragments were washed with PBS (phosphate-buffered saline) and stained with a methanol-crystal violet solution ( $0.4 \%$; wt $/ \mathrm{vol})$. Bones from non-5FU-treated mice were processed as a control for osteoblast growth recovery.

\section{Cell culture}

Primary osteoblasts were seeded in a 12-well plate. To investigate the effect of FGF2 in primary osteoblasts from 5FU-treated mice, FGF2 (50 or $80 \mathrm{ng} / \mathrm{ml}$ ) was added to cultured primary osteoblasts for 15 days. 
The FGFR inhibitor SU5402 (Sigma-Aldrich) was added to the culture medium at a final concentration of $5 \mu \mathrm{M}$.

\section{RNA isolation and real-time RT-PCR}

Total RNA was isolated from primary osteoblasts using TRIzol reagent (Invitrogen, Carlsbad, CA) according to the manufacturer's protocol. Total RNA $(1-1.5 \mu \mathrm{g})$ was reverse-transcribed using the Omniscript Reverse Transcription kit (QIAGEN, Valencia, CA). mRNA expression levels were measured either by conventional PCR or by fluorescence-based real-time PCR. Quantitative real-time PCR using CFX Connect ${ }^{\mathrm{tm}}$ Real-Time PCR system (Bio-Rad, Hercules, CA) or conventional PCR using Go-taq ${ }^{\circ}$ Flexi DNA polymerase (Promega, Madison, WI) was performed in a volume of $15 \mu \mathrm{l} 1 \times$ SYBR $^{\circ}$ green premix Ex Taq ${ }^{\text {Tim }}$ (TAKARA BIO Inc., Shinga, Japan) and $20 \mathrm{pM}$ forward and reverse primers (Bioneer, Daejeon, Republic of Korea). To normalize for input RNA, murine Gapdh (Glyceraldehyde-3-phosphate dehydrogenase) was amplified as an endogenous control. Amplification conditions were as follows: $95{ }^{\circ} \mathrm{C}$ for 5 mins followed by 40 cycles of $95{ }^{\circ} \mathrm{C}$ for $20 \mathrm{~s}$, and annealing temperature of each primer for $30 \mathrm{~s}$. The melting curve protocol was performed for each primer set to confirm specificity. All samples were run in triplicate. The primer sequences for each gene are shown in Table 1.

\section{Western blotting}

Western blot analysis was performed as previously reported [21]. Briefly, primary osteoblasts isolated from PBS- or 5FU treated mice were lysed in lysis buffer (RIPA buffer, $50 \mathrm{mM}$ Tris-Cl [pH 8.0], $150 \mathrm{mM} \mathrm{NaCl}$, 1\% NP-40, 5 mM EDTA, 1 mM PMSF; Thermo Scientific, Rockford, IL) containing a protease cocktail (Roche, Mannheim, Germany) and phosphatase cocktail. Equal amounts of cell lysate protein were subjected to SDSPAGE and transferred to nitrocellulose membranes (Whatman, GE healthcare Bioscience, Pittsburgh, PA). After blocking, the membranes were incubated with primary antibodies (phospho-p44/p42 MAPK (Erk1/2) (1:3000) (Cell Signaling, Beverly, MA) and ERK (1:500) (Santa Cruz Biotechnology, Inc., Dallas, TX), overnight at $4{ }^{\circ} \mathrm{C}$ and then incubated with the appropriate horseradish peroxidase-conjugated secondary antibody for $1 \mathrm{~h}$ at RT. The blots were developed using a chemiluminescence detection system (ECL kit; Amersham Pharmacia Biotech, Piscataway, NJ) and exposed to $\mathrm{x}$-ray film (AGFA, Mortsel, Belgium).

\section{Harris hematoxylin and eosin staining}

The femora and tibiae of PBS- or 5FU- treated mice were fixed in $4 \%$ paraformaldehyde phosphate buffer solution (Wako, Osaka, Japan). Bones were decalcified in 0.5 M EDTA (ethylenediaminetetraacetic acid) (Welgene, Daejeon, Republic of Korea) for 3 weeks before dehydration, paraffin-embedded, and cut into 5- to 7- $\mu \mathrm{m}$

Table 1 Gene primers list

\begin{tabular}{|c|c|c|}
\hline \multirow[t]{2}{*}{ Target gene } & \multicolumn{2}{|l|}{ Primer sequence } \\
\hline & Forward (5'-3') & Reverse $\left(5^{\prime}-3^{\prime}\right)$ \\
\hline Gapdh & GGCATTGCTCTCAATGACAA & TGTGAGGGAGATGCTCAGTG \\
\hline Alp & GATAACGAGATGCCACCAGAGG & TCCACGTCGGTTCTGTTCTTC \\
\hline Runx2 & AGATGATGACACTGCCACCTCTG & GGGATGAAATGCTTGGGAACTGC \\
\hline Opn & TGCACCCAGATCCTATAGCC & CTCCATCGTCATCATCATCG \\
\hline Osx & СTCTCTGCTTGAGGAAGAAG & GTCCATTGGTGCTTGAGAAG \\
\hline Ki67 & CAGACGAGCAAGAGACAAAG & AAGTACGGAGCCTGTATCAC \\
\hline SDF-1 & GCTCTGCATCAGTGACGGTA & TAATTTCGGGTCAATGCACA \\
\hline Jagged-1 & CTTCAATCTCAAGGCCAGCC & CAGGCGAAACTGAAAGGCAG \\
\hline$N$-cadherin & ACGAGAGGCCTATCCATGCTGAGC & TCAGTCGTCACCACCGCCGTACAT \\
\hline Vcam-1 & ATTTCTGGGGCAGGAAGTT & ACGTCAGAACAACCGAATCC \\
\hline Angpt1 & AGGCTTGGTTTCTCGTCAGA & TCTGCACAGTCTCGAAATGG \\
\hline Fgfrilllb & CTTGACGTCGTGGAACGATCT & AGA ACGGTCAACCATGCAGAG \\
\hline Fgfrillle & AGAGACCAGCTGTGATGACC & TGTTACCTGTCTGCGCAGAG \\
\hline Fgfr2lllb & CCCATCCTCCAAGCTGGACTG & CAGAGCCAGCACTTCTGCATTG \\
\hline Fgfrallle & CCCATCCTCCAAGCTGGACTG & TCTCACAGGCGCTGGCAGAAC \\
\hline Fgfr3lllb & GGCGCTAACACCACCGAC & TGGCAGCACCACCAGCCAC \\
\hline Fgfr3llle & TGGAAACTGATGAGGCTGGC & AAACGAGAGACCTTGTGCAC \\
\hline
\end{tabular}


sections. For osteoblast analysis in 5FU treated mice, sections were stained with Harris hematoxylin and eosin (Sigma-Aldrich).

\section{RNA-sequencing analysis}

Total RNA was isolated for analyze RNA-sequencing using RNeasy PLUS Micro (QIAGEN) according to the manufacturer's protocol. The RNA-sequencing library was prepared using an Illumina TruSeq RNA sample prep kit and quantified using a KAPA library quantification kit. Total library fragments were used to generate clusters, followed by sequencing on an Illumina HiSeq 2500. Gene expression was quantitated using Cufflinks v2.1.1.

\section{Immunohistochemical analysis}

For immunohistochemistry analysis, the bone tissue (femur and tibiae) were harvested from PBS- and 5-FUtreated mice on 3 days and 6 days and processed for immunohistochemistry [22]. Tibiae were fixed in $4 \%$ paraformaldehyde (PFA) and paraffin-embedded and cut into 6- $\mu \mathrm{m}$ sections. After dewaxing and rehydrating, endogenous peroxidase was quenched for $15 \mathrm{~min}$ with $3 \%$ $\mathrm{H}_{2} \mathrm{O}_{2}$ in methanol. Heat-mediated antigen retrieval and enzymatic techniques were performed according to recommendations for the specific antibodies. A blocking step was performed using $10 \%$ normal goat serum and $1 \%$ bovine serum albumin (BSA) in PBS. After endogenous peroxidase and nonspecific protein block, primary antibodies were incubated overnight at $4{ }^{\circ} \mathrm{C}$ as follows: anti-FGF2 (Santa Cruz, CA, USA) primary antibodies. Polyclonal secondary antibodies were incubated, followed by incubation in streptavidin horseradish peroxidase (Invitrogen, CA, USA). Staining was developed with $\mathrm{DAB}$ according to manufacturer's instructions (Invitrogen, CA, USA) and briefly counterstained in methy green before coverslipping in cytoseal permanent mounting media. Localization of positive staining was analyzed by light microscopy (Olympus CX41, Japan).

\section{Statistical analyses}

Data are presented as means $\pm \mathrm{SD}$, and statistical comparisons between groups were performed by oneway analysis of ANOVA test. * and * symbols indicate $p<0.05$ and $p<0.01$ vs control group, respectively.

\section{Results}

\section{Bone marrow suppression increases HSC niche}

\section{related-genes in osteoblasts}

The 5FU treatment eliminates actively cycling cells in the BM and induces surviving quiescent LT-HSC activation. We first investigated whether the osteoblastic niche in 5FU-treated mice could change to support HSC reconstitution in response to this BM damage. After administering a sublethal dose of $5 \mathrm{FU}$ to the mice, we observed an increase in HSC niche-related gene expression in primary osteoblasts isolated from mouse long bones (femurs, tibiae and humeri). HSC niche-related genes, N-cad, Jagged-1, Angpt1, Vcam-1, Scf, and sdf-1 significantly increased in the osteoblasts isolated from 5FU-treated mice for 4 days compared to control osteoblasts (Fig. 1a). However, the proliferation marker gene Ki67 was highly decreased in the OB after 4 days of 5FU treatment (Fig. 1b). It was previously shown that osteocalcin- and collagen I-positive cells increased in trabecular bones of the metaphysis of irradiated mice, and the mRNA expression of niche marker genes increased in endosteal osteoblasts isolated from irradiated mice [16]. These results suggest that the osteoblast lineage cells at the endosteal surface change to be activated as niche cells after 5FU-induced BM damage.

\section{Bone marrow suppression also increases osteoblast marker genes expression}

Since it is known that early stage osteoblast cells serve as HSC niche cells [23], we also examined whether osteoblast marker genes are increased upon 5FU treatment. It was previously shown that endosteal osteoblasts of irradiated mice increased osteocalcin and collagen type I expression and that hematopoietic cell clusters closely contacted to the bone (endosteal osteoblast zone) after 5FU treatment [16, 24]. In our study, the early osteoblast marker genes Runx2 and $O s x$ were increased in osteoblasts of 5FU-treated mice compared to control (Fig. 1c and d). Alp and Opn expressions were also increased (Fig. 1e and f). These data suggest that 5FU-induced BM damage leads to the activation of niche cells at early stage of osteoblast in the BM.

\section{FU-damaged bone marrow induces decreased proliferation rates of endosteal osteoblasts}

To determine whether 5FU treatment affected endosteal osteoblast survival and proliferation, we maintained OBs selectively isolated from long bone explants of 5FUtreated mice in vitro. Figure $2 \mathrm{a}$ and $\mathrm{b}$ show photographs of in vitro-cultured OBs for 2 weeks from the bone of PBS- or 5FU-treated mice for 4 days. OBs obtained from 5FU-treated mice showed a reduction in proliferation rate compared to the control. We also counted OB colonies at 2 weeks by CFU-F assay and measured the O.D. value of OB colonies from 5FU-treated mice and control mice (Fig. 2c and d). 5FU treatment-induced bone marrow suppression not only eliminated actively cycling cells in the $\mathrm{BM}$ but also affected osteoblast proliferation.

Fibroblast growth factor 2 supports the proliferation rate and increases HSC niche-related genes expression in endosteal osteoblasts of 5FU-treated mice.

We have shown that acute injury by $5 F U$ to suppress bone marrow cycling cells also affected the endosteal 

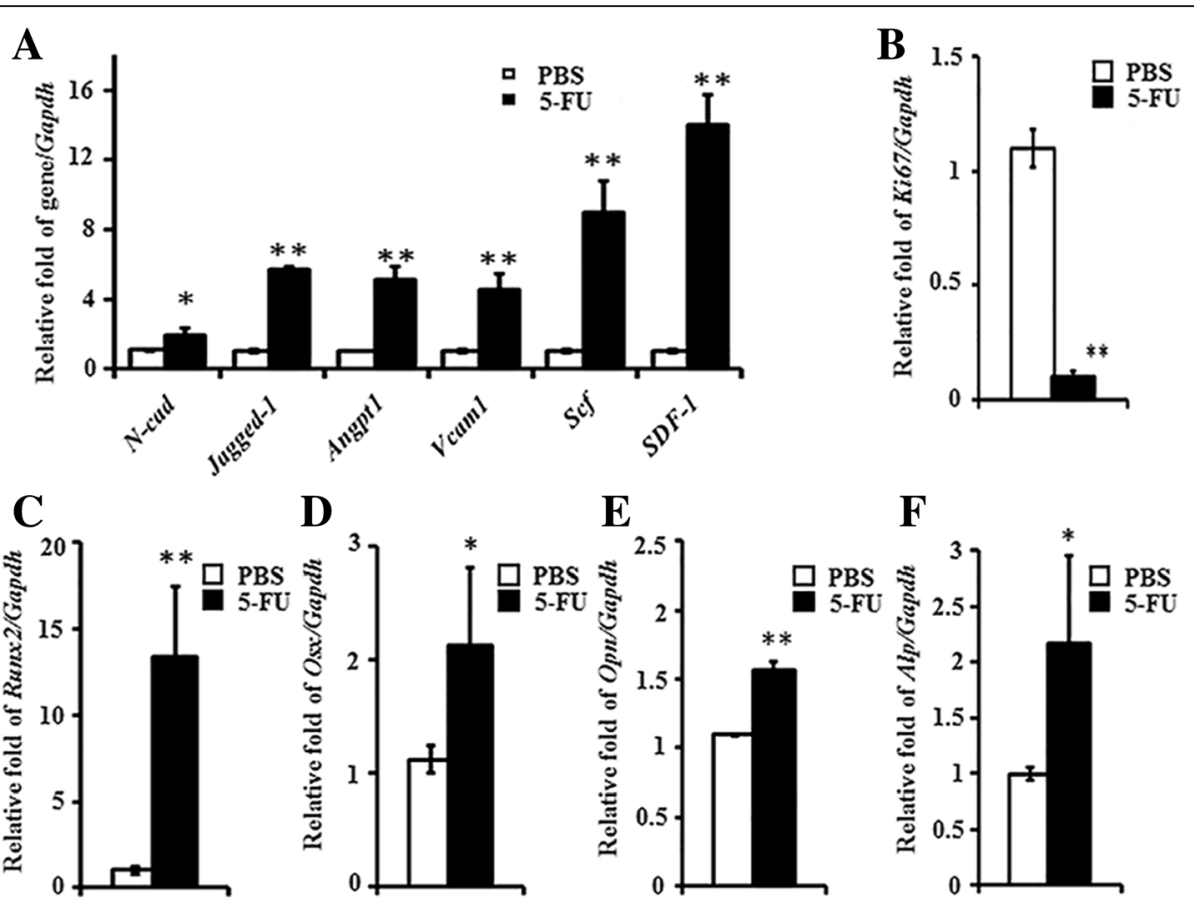

$\mathbf{E}$

Fig. $1 \mathrm{HSC}$ niche related-genes and bone marker genes in primary endosteal osteoblasts isolated from 5FU-treated mice. Total RNA was isolated from the osteoblasts of the mice treated with PBS $(n=5)$ or $5 F U(n=5)$ for 4 days. a, b The mRNA expression levels of SDF-1, N-cad, Jagged-1, Angpt1, Vcam 1, Scf (a) and Ki67 (b) related to Gapdh levels were analyzed by qRT-PCR and presented as ratios to the control (PBS) OBs' relative value. c-f The mRNA expression levels of Runx2 (a), Osx (b), Opn (c) and Alp (d) related to Gapdh levels were analyzed by qRT-PCR and presented as ratios to the control (PBS)

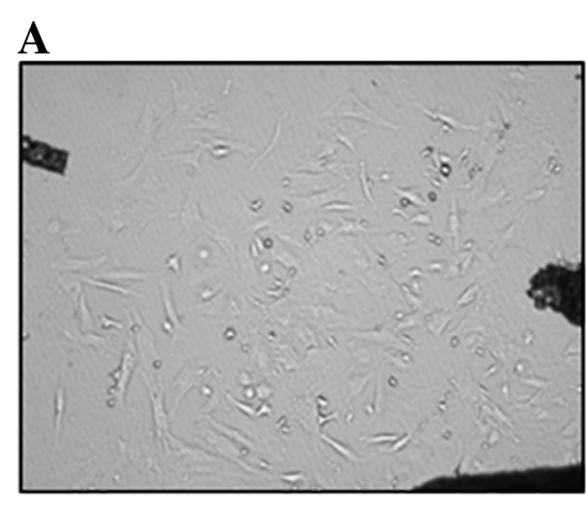

C

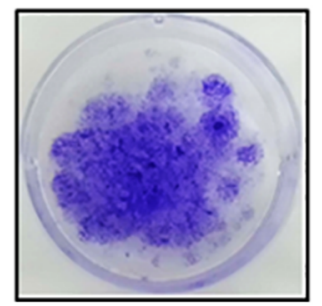

B

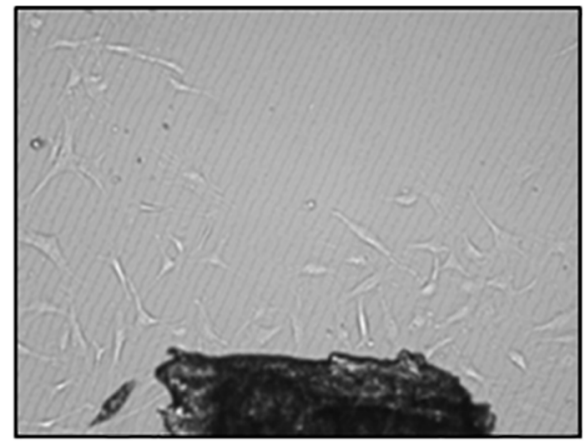

D

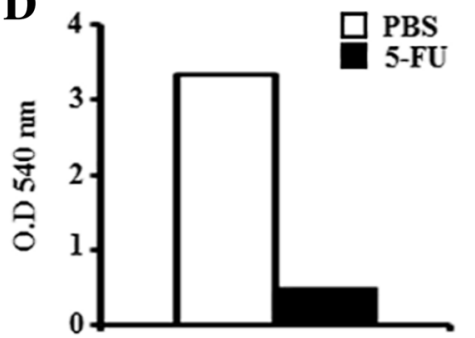

Fig. 2 Proliferation rate of endosteal osteoblasts isolated from 5FU-treated mice. Representative photographs of in vitro-cultured OBs selectively obtained from bones in PBS- (a) or 5FU-treated mice (b) for 4 days $(n=5)$. Long bone fragments completely depleted of bone marrow were cultured in a-MEM medium containing 15\% FBS for 14 days. c \& d Representative pictures of OB colonies from PBS- or 5FU-treated mice after 14 days of culture CFU assay (c), and O.D. values at $540 \mathrm{~nm}$ (per $44 \mathrm{mg}$ of processed bone chip) after 2 weeks of culture of OBs from PBS- or 5FU-treated mice (d) 
osteoblastic niche cell proliferation ex vivo (Fig. 2). Therefore, we hypothesized that endosteal osteoblastic niche cells should be activated by various factors, including cytokines and growth factors for the niche cells to be activated to support HSPCs and to allow for damaged $\mathrm{BM}$ to recover after acute injury. Recently, mature megakaryocytes (MMs) residing within BM have been shown to directly and indirectly influence various cells in the $\mathrm{BM}$, including HSCs and OBs. MMs stimulate their proliferation and differentiation through the regulation of OB function [25-27]. MMs are known to be a major source of factors, including insulin-like growth factor (IGF) -1 and fibroblast growth factor 2 (FGF2), that are important in $\mathrm{OB}$ regulation and recovery in damaged BM $[9,15,16]$. We investigated the effect of FGF2 on osteoblastic niche cell proliferation and function in endosteal OBs from 5FU-treated mice. The primary cells from the mice were cultured for 2 weeks with or without FGF2 (50 ng/ml) and OB colonies were examined by crystal violet staining and measured the O.D. value of the colonies in cultured OBs from 5FU-treated mice and control. The colony formation in CFU-F assay and the O.D. values (normalized to the bone weight used for primary cell culture and compared to 'without FGF2' control (O.D. $540 \mathrm{~nm} /$ control)) were significantly increased (126-fold) in FGF2-treated OBs from 5FUtreated mice while the colonies were mildly increased in PBS control OBs by FGF2 treatment (Fig. 3a and b).

The expressions of HSC niche-related genes, Scf, Jagged-1 and N-cadherin, were increased in FGF2 $(50 \mathrm{ng} / \mathrm{ml})$-treated OBs (Fig. 3c-e). However, although $N$-cad mRNA expression was significantly increased in $50 \mathrm{ng} / \mathrm{ml}$ FGF2 treatment (Fig. 3e), Jagged-1 level was decreased by high concentration of FGF2 (80 ng/ml) (Fig. 3d). In addition, FGFR inhibitor SU5402 (5 $\mu \mathrm{M})$ treatment resulted in the reduction of these nicherelated $S c f$, Jagged-1, and $N$-cadherin gene expression from the increase of FGF2 to almost basal levels
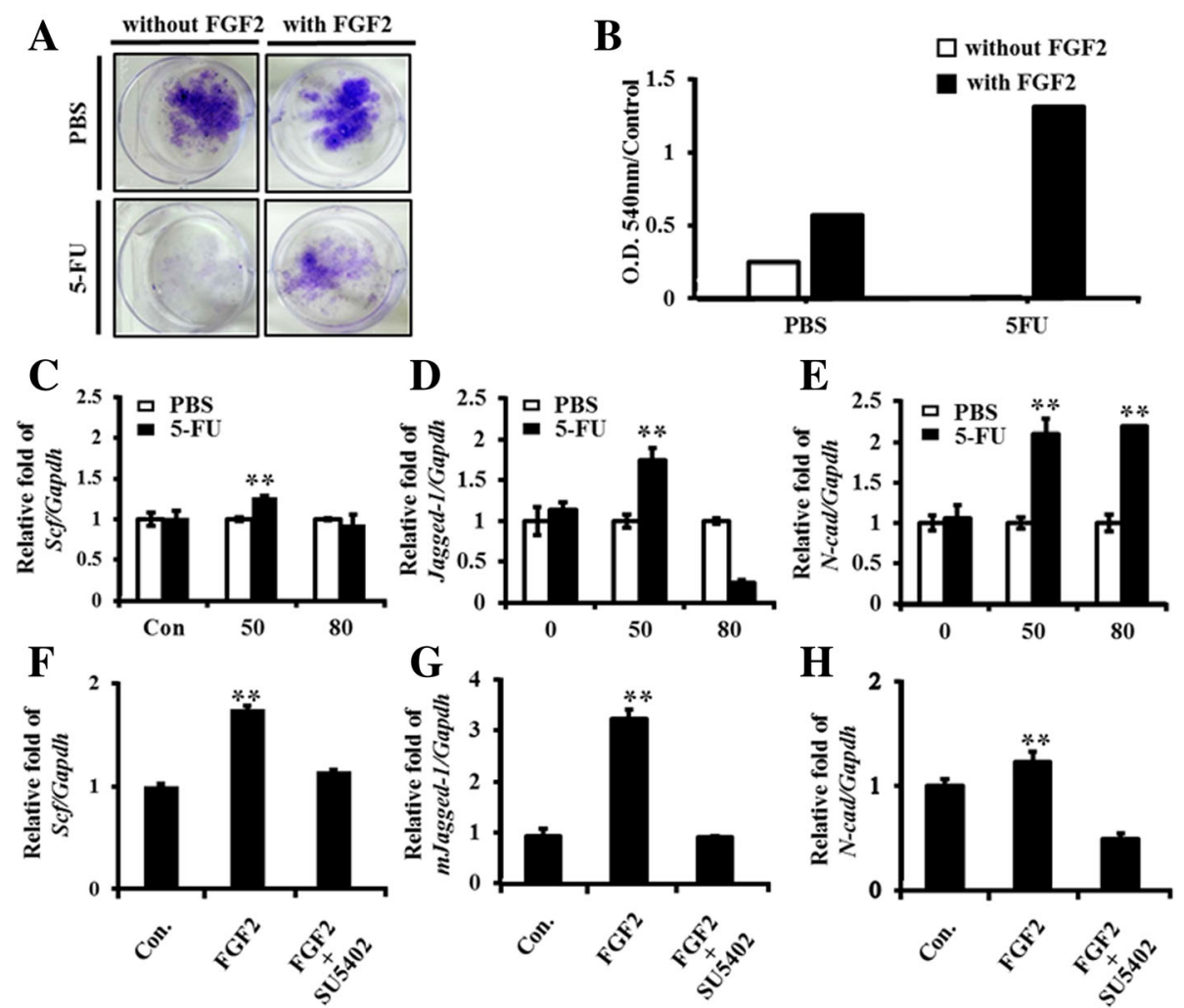

Fig. 3 The proliferation rate and expression of HSC niche-related genes increased by FGF2 in endosteal osteoblasts isolated from 5FU-treated mice. $\mathbf{a}$ \& $\mathbf{b}$ Representative pictures of OB colonies from PBS- or 5FU-treated mice for 4 days, after culture in the presence or absence of FGF2 $(50 \mathrm{ng} / \mathrm{ml})$ for 14 days. CFU assay was performed and O.D. values were measured at $540 \mathrm{~nm}$. The O.D value was normalized to the bone weight used for primary cell culture and was presented as a relative O.D. values to the control of 'without FGF2' (thus expressed 'O.D. 540 nm/control') (c, d, e) The PBS- or 5FU-activated OBs were cultured in the presence of FGF2 at various concentrations (0, 50, 80 ng/ml) for 14 days. Total RNA was isolated, and the expression of HSC niche-related genes Scf, Jagged-1, N-cad was analyzed compared to Gapdh levels by qRT-PCR and presented as ratios to the control (PBS) OBs' relative value. $\mathbf{f}, \mathbf{g}, \mathbf{h}$ Blocking of FGF receptor signaling by FGFR inhibitor. 5FU-activated OBs were cultured from 5FU-treated mice for 4 days in the absence or presence of SU5402 (5 $\mu \mathrm{M})$ for 14 days. Total RNA was isolated and the expression of HSC niche-related genes Scf (f), Jagged-1 (g), and $\mathrm{N}$-cad (h) were analyzed against Gapdh levels by qRT-PCR and presented as ratios to the control OBs' relative value 
(Fig. 3f-h). These data highlight the importance of FGF2 signaling in the regulation of HSC nicherelated genes upon BM suppression.

\section{Fgfrs subtype expression in endosteal osteoblasts}

We have shown that FGF2 supports osteoblastic niche cells, and increases HSC niche-related gene expression and the proliferation rate of OBs from 5FU-treated mice to allow for the recovery of BM suppression. To confirm FGF signaling activation in the endosteal OBs under stress, we performed PCR analysis to examine Fgfr expression in the endosteal OBs. The endosteal OBs expressed Fgfr1-3, including all subtypes except Fgfr2IIIb and Fgfr4 (Fig. 4). Surprisingly, the expressions of Fgfr1IIIb, Fgfr2IIIb, Fgfr2IIIc, and Fgfr3IIIb were

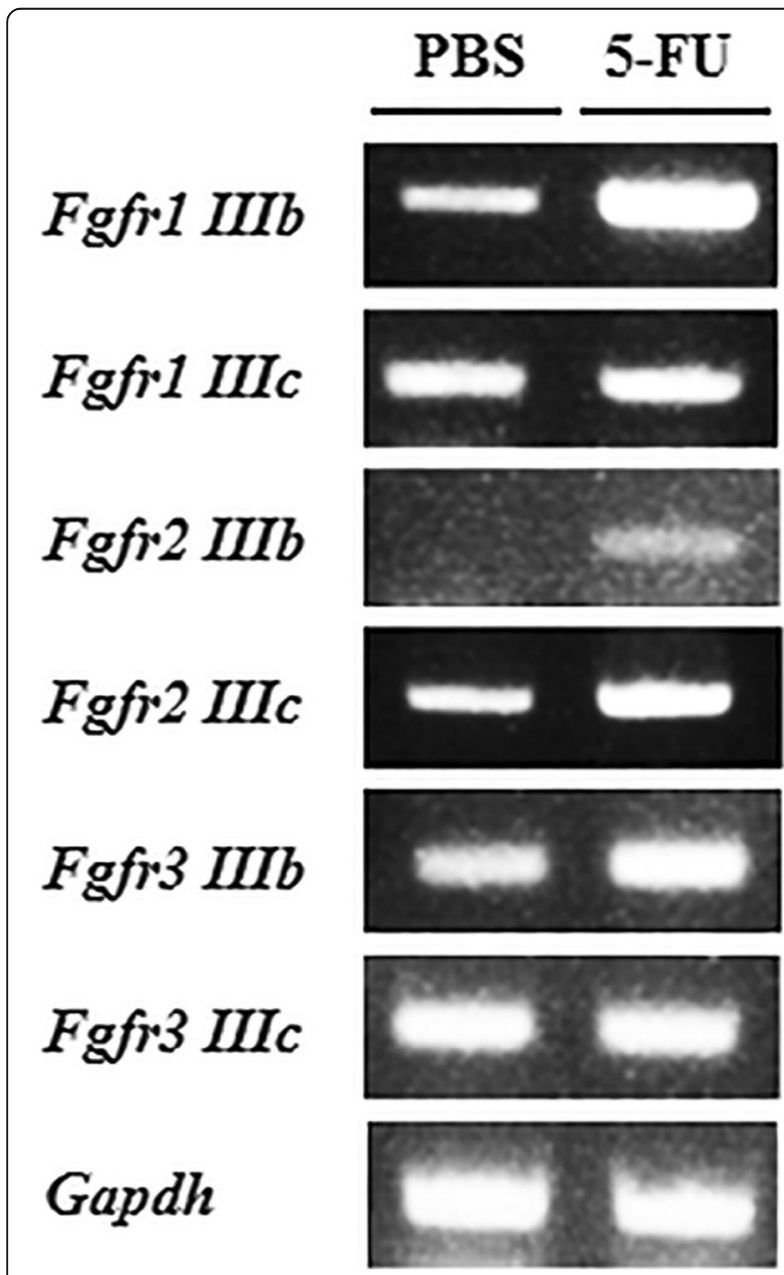

Fig. 4 Expression of Fgfr genes in the endosteal osteoblasts isolated from 5FU-treated mice. Total RNA from PBS- or 5FU-activated OBs for 4 days was subjected to RT-PCR. Expression of Fgfr genes in these cells was confirmed using primer pairs by PCR. PCR products were separated on a $1.5 \%$ agarose gel containing ethidium bromide (EtBr) and visualized under UV light. Representative results from at least 3 independent experiments are shown increased after 5FU stress. These results suggest that Fgfrs were activated to receive more FGFs signal in the OBs of 5FU-treated mice.

\section{Expression of Fgfrs and the proliferation marker gene $K i 67$ in the osteoprogenitor (osterix-positive) cells of 5FU-treated mice}

The BM environment is composed of multiple cell types, including endosteal osteoblastic niche cells and osteolineage cells, which support HSPC homeostasis. Osteoblast progenitor and mesenchymal stem cells highly express the cytokines SDF-1 and Scf, which are critical for HSC maintenance in mice $[19,28]$. Osx-positive osteoprogenitor cells are important osteoblastic niche cells, although Osx-expressing OBs are restricted short-lived cells in the adult BM [29]. We sorted osteoprogenitor (GFP-positive) cells by FACS from PBS- or 5FUtreated Os $x$-GFP-Cre mice for 4 days to perform RNA-seq (Fig. 5a). Approximately 6000 to 10,000 GFP positive cells were collected from about 20 mice in each group of either control or 5FU activated BMs of Osx-GFP-Cre mice. These GFP+ cells in 5FU treated mice expressed the HSC niche-related genes Angpt1, Vcam1, Sdf-1, and Scf higher than the control group (Fig. 5b). RNA-seq analysis on sorted Osx + cells showed FPKMs (fragments per kilobase of exon per million fragments mapped) for Fgfr 1, Fgfr2, and Fgfr3 were very high in 5FU-Osx-positive cells compared to PBS control (Fig. 5c). Among the 3 Fgfrs, Fgfr2 was more highly expressed than Fgfr1 and Fgfr3. The qRT-PCR of Fgfr $1-3$ expressions in Osx-positive cells from 5FU-treated mice after BM suppression showed that Fgfr1 (10.23-fold), Fgfr2 (448.8-fold), and Fgfr3 (114.52-fold) were significantly increased in Osx-positive cells (Fig. 5d-f). Ki67 expression also increased (Fig. 5g). Increased Fgfr 1-3 and Ki67 expression indicates that early stage Osx-positive osteoblastic niche cells become highly sensitive to FGF signaling by increasing their FGF receptors and proliferate after BM stress.

\section{Endosteal osteoblasts activated the ERK pathway and osteoblast expansion after BM suppression by $5 \mathrm{FU}$ treatment}

To test whether the FGF2 levels are affected during the early recovery phase after $5 \mathrm{FU}$ treatment, bone marrow tissues were immunostained with anti-FGF2 antibody. Three days after 5FU treatment, protein levels of FGF2 in bone marrow was significantly increased in $5 \mathrm{FU}$ treatment compared to PBS control. The staining shows a saturated level of FGF2 in 5FU treated 3-day sample (Fig. 6a). To confirm also whether FGF2 downstream signaling is activated in endosteal OBs following 5FU treatment, phospho-ERK, a downstream of Fgfr activation, was measured by western blot. The phospho-ERK 
A

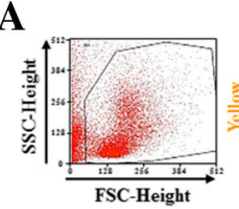

OSX-GFP

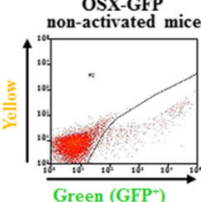

D

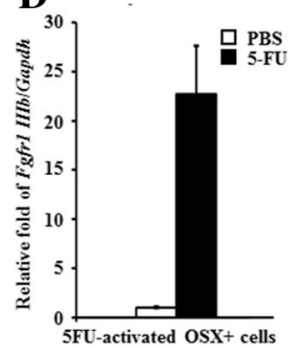

WT; non-activated



OSX-GFP

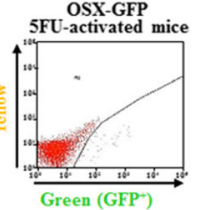

$\mathbf{E}$



$\mathbf{F}$
C

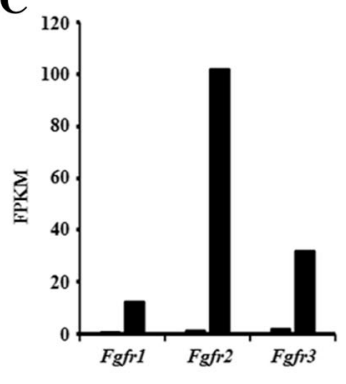

G

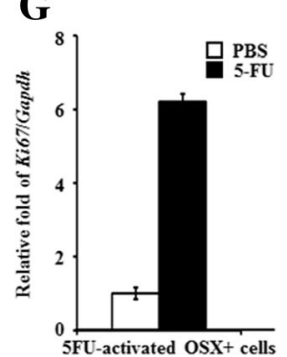

Fig. 5 FGF signal and HSC niche-related genes were activated in osteoprogenitor cells (OsX+ cells) isolated from 5FU-treated Osx-GFP mice. a FACS analysis to sort GFP-positive cells from Osx-GFP mouse treated with 5FU for 4 days. b Total RNA was isolated from PBS- or 5FU-activated Osx positive cells (osteoprogenitor cells). mRNA expression levels of SDF-1, Angpt1, Vcam1 and Scf related to Gapdh levels were analyzed by qRT-PCR and presented as ratios to the control (PBS) Osx positive cells' relative value. c RNA-seq analysis of PBS- or 5FU-activated Osx+ cells for Fgfrs. Expression level shown by FPKM (Fragments per kilobase of exon per million fragments mapped). d, e, $\mathbf{f}$ Gene expression of Fgfr1-3 using qRT-PCR of Osx+ osteoprogenitor cells from PBS- or 5FU-treated mice for 4 days. $\mathbf{g}$ Expression of Ki67 gene in Osx+ progenitor cells from PBS- and 5FU-treated mice for 4 days
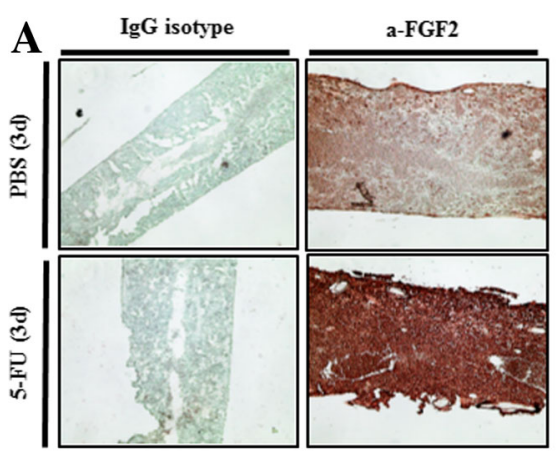

B

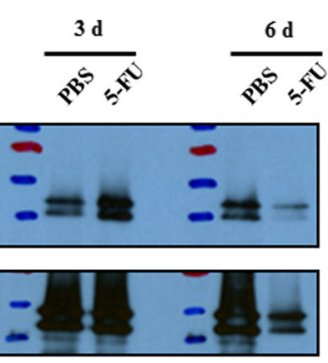

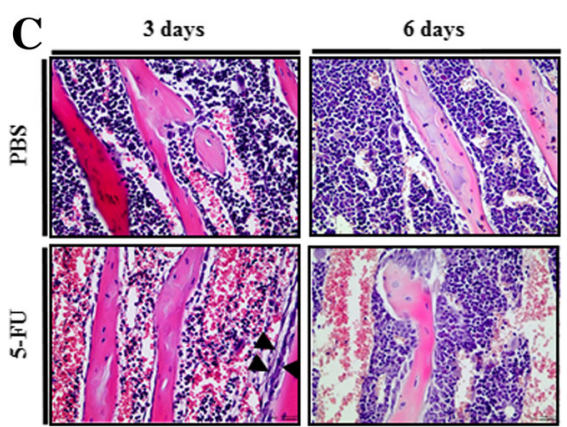

Fig. 6 FGF2 increase, ERK pathway activation, and the expansion of endosteal osteoblast population following 5FU treatment. a Immunohistochemical staining on the bone marrow of mice 3 days after $5 F U$ treatment. Bone section counterstained with methyl green. Magnification $\times 100$. $\mathbf{b}$ Total proteins were extracted at 3 and 6 days after 5FU treatment in endosteal OBs from PBS- or 5FU-treated mice. pERK and ERK expression was analyzed by western blotting. c Representative H\&E stained sections of tibiae at 3 and 6 days following 5FU treatment in mice. Endosteal OBs showed increased cellularity along the endosteal surface at the indicated time points. Arrows indicates multilayer cuboidal osteoblast on the trabecular bone 
protein levels in endosteal $\mathrm{OBs}$ isolated from 5FUtreated mice at 3 days highly increased, (Fig. 6b). These results suggest that $\mathrm{BM}$ suppression by $5 \mathrm{FU}$ treatment induced these acute injury and FGF2 increase followed by Fgfr-pERK activation in osteoblastic niche cells lasts 3-4 days. Thereafter, pERK signals decrease. Short-term increases of FGF proteins in the BM after 5FU treatment could support osteoblastic niche cells to recover HSPC through FGF signaling.

It was previously shown that osteoblast expansion increased $48 \mathrm{~h}$ after radioablative conditions [17]. We investigated whether osteoblast expansion increased to support HSPCs during short-term recovery in the BM after 5FU treatment in mice. Histological analysis showed endosteal osteoblast expansion from a single layer in control (PBS-treated) mice to several layers at 3 days in 5FU-treated mice (Fig. 6c). Expanded endosteal OBs also showed changed morphology from flattened to cuboidal. This morphological changes may increase adhesive interactions between the osteoblastic niche and HSPCs, and migration [30].

\section{Discussion}

The BM and HSC niche support HSC self-renewal and differentiation through interactions of various BM cells and secreted molecule such as FGFs and EGF $[9,31,32]$. The majority of HSCs are quiescent during homeostasis, but these cells can be activated to proliferate and differentiate in response to infectious stress [33]. High dose chemotherapy or radiation treatment for hematological disease such as leukemia or lymphoma may induce damage to the BM HSC niche. Chemotherapy also affects HSC niche cells, such as osteoprogenitor cells, endothelial cells, and megakaryocytes, and induces changes to make an appropriate microenvironment for HSC regeneration [5].

Our data indicate that FGF signaling is important in osteoblastic niche cells, including Osx-positive osteoprogenitor cells, in the recovery following 5FU-induced acute bone injury. These conclusions are based on evidences that (1) HSC niche-related gene expression increased in osteoblastic niche cells isolated from 5FUtreated mice, (2) FGF2 increased proliferation in damaged osteoblastic niche cells, and (3) FGF receptor signaling increased in osteoblastic niche cells, especially Osx + osteoprogenitor cells, following 5FU treatment.

A fundamental question in our studies was whether increased FGFs in the BM following 5FU could recover osteoblastic niche cells to support regeneration of BM suppression. The proliferation marker Ki67 decreased after 5FU treatment at 4 days and also osteoblastic niche cell proliferation was inhibited in vitro during cell culture (Figs. 1 and 2). However, OB cell proliferation was increased dramatically and niche markers were also increased upon FGF2 treatment in the BM cells from 5FU stressed mice (Fig. 3). It is also of note that recovery phases after 4 days increased the levels of FGF protein in damaged BM and the proliferation of osteoblastic cells in damaged bone through radiation treatment $[9,16]$. We also confirmed that the osteoblast marker genes Runx2, Osx, Opn, and Alp increased in osteoblastic niche cells at 4 days following $5 \mathrm{FU}$ (Fig. 1). In particular, the early osteoblastic markers Runx2 and Osx increased in osteoblasts from 5FU-treated mice. These results corresponded with amplified cellularity along the endosteal surface possibly from endogenous osteoprogenitor cells 3 days after BM suppression (Fig. 6c).

To address the mechanisms of OB survival and proliferation in $\mathrm{BM}$ after $5 \mathrm{FU}$ treatment in mice, we focused on FGF2, a factor that induces an increase in the OB population and promotes early $\mathrm{OB}$ differentiation, because it is known that FGF2 is highly secreted in the BM of 5FU-treated BM-suppressed mice [34, 35]. OBs from 5FU-treated mice significantly reduced the proliferation rate (Fig. 2). These results also indicate that OBs were initially damaged in the $\mathrm{BM}$ following $5 \mathrm{FU}$, as shown by decreased Ki67 expression. Conversely, FGF2 treatment induced the proliferation of OBs damaged by $5 \mathrm{FU}$ during in vitro culture (Fig. 3a and b). It also induced increased HSC niche-related gene expression, including Scf, Jagged-1, and N-cad (Fig. 3c-e). N-cad was highly expressed in the endosteal $\mathrm{ALCAM}^{+} \mathrm{SCA}-1^{-}$cell population, which can enhance LTR (long-term reconstituting) activity [36]. Jagged-1 expression enhances HSPC selfrenewal capacity $[37,38]$. Recently, FGF2 was shown to expand stromal cells that may promote HSPC expansion by increasing $S C F$ [13]. These results showed that FGF2 could induce niche-related gene expression and increase niche cell proliferation to support HSPCs in 5FUdamaged osteoblastic niche cells.

FGFR inhibition through the FGFR antagonist SU5402 affected HSC niche-related gene expression, including Scf, Jagged-1, and N-cad (Fig. 3f-h). We confirmed the expression of Fgfrs compared to controls in osteoblastic niche cells of mice following 5FU treatment (Fig. 4). Fgfr1, Fgfr2 and Fgfr3 were highly expressed in 5FUtreated endosteal OBs with exception of IIIc form of Fgfr1 and Fgfr3. FGFR1 and FGFR2 expression in OBs have been extensively characterized. Importantly, FGFR1/2 signaling controls osteoblast gene expression and bone formation $[18,39]$. Our results also indicate that Fgfr IIIb forms and FgF2 IIIC might play important roles in the reception of FGF2 signals activated by $5 \mathrm{FU}$ induced bone marrow suppression. We confirmed FGF signaling activation by pERK protein levels in the endosteal OBs isolated from 5FU-treated mice at the early 3-day time point (Fig. 6b). All together these results indicate that pERK activation through Fgf receptor upregulation 
increases endosteal $\mathrm{OB}$ proliferation after 5FU-treated $\mathrm{OB}$ cells received higher signal from bone marrow.

The function of OBs in proliferation and differentiation is known to relate to the HSPC supportive microenvironment [37, 40, 41]. HSC-niche factors, such as SDF-1/CXCL12 and Scf, in osteoblast lineage cells or other HSC niche cells differentially regulate HSC maintenance $[19,42]$. Here, we focused on the effect of FGF2 in osteoblast, a HSC niche, recovery, and specifically the activity of endosteal osteoblastic niche cells, which showed expansion of $\mathrm{OB}$ layers in trabecular bone of BM following 5-FU treatment (Fig. 6c). We hypothesize that endogenous osteoprogenitor cells after BM suppression increase and then cause amplified cellularity along the endosteal surface. These amplified OBs could provide HSPC with a supportive microenvironment. It was reported that FGF2 modulates Nestin-positive mesenchymal stem cells and MS-5 murine stromal cells by generating high levels of Scf. Also, the number of BM HSPCs decreased in FGF2 KO mice. Mice treated with FGF2 affected trabecular bone growth and formation [13]. The Notch ligand Jagged-1 has been reported to increase bone formation via parathyroid hormone (PTH)dependent $\mathrm{PKC}$ pathway in OBs and has important roles in stromal interactions with HSCs [37, 43]. FGF2 could maximize the anabolic effect of PTH, because FGFR signaling is also coupled to PKC pathway [18].

Since the endosteal OBs are a heterogeneous population, we examined whether the resident osteoprogenitor cells in 5FU-treated mice activated FGF signaling by increasing FGF protein levels. Conditional deletion of Osx in bone almost eliminated hematopoiesis in the BM metaphysis, and suppressing Osx expression inhibited niche generation $[20,44]$. It seems that Osx-positive progenitor cells play an important role in HSC niche generation. To confirm this hypothesis, we sorted-Osx positive osteoprogenitor cells from PBS or 5FU-treated Osx-GFP mice by FACS and then performed RNASequencing. HSC niche-related genes increased in the Osx + cells isolated from 5FU-treated Osx-GFP mice (Fig. 5b). Angpt1 and Scf expression significantly increased following 5FU treatment. The results of RNA-Seq analysis showed that Fgfr $1-3$ expression was significantly detected in 5FU-treated Osx + cells, but not Fgfr4 (Fig. 5c-f). Ki67 expression in Osx-positive cells from 5FU-treated mice increased at day 4 (Fig. 5g). These results indicate that the Osx + resident osteoprogenitor cells in BM can activate FGF signaling in mice under stress conditions. Similar up-regulation of Fgfr was reported in Nestin + MSCs in which the number of Nestin + MSCs that express Fgfr1 and Fgfr2 in FGF2-administered mice increased [13]. It is suggested that Osx + cells might support HSCs to allow for recovery of BM suppression via FGF signaling under stressed conditions. Scf, a downstream factor of FGF2, was also significantly expressed in Osx + cells following $5 \mathrm{FU}$ treatment.

\section{Conclusions}

Our results indicate an important role for FGF2 in supporting HSC maintenance in stress-induced osteoblastic niche cell expansion. 5FU-treated mice induced BM suppression and showed inhibited osteoblastic niche cell proliferation with decreased Ki67. However, FGF2 could induce the proliferation of OBs, which express Fgfrl-3 and highly express HSC niche-related genes during in vitro culture. FGF signaling was activated via increased pERK protein in the endosteal osteoblastic niche cells during the recovery phase. In mouse BM following 5FU, we confirmed the expansion of the $\mathrm{OB}$ layer in trabecular bone. HSC-niche related genes and Fgfr1-3 were also highly expressed in Osx + osteoprogenitor cells under stress, suggesting close relation of Osx + cells in the recovery phase. In the 5FU-induced stress situation, FGF signaling could support endosteal osteoblastic niche cell proliferation as well as the reinforcement of HSC niche factors to allow for the recovery from BM HSC suppression.

\section{Abbreviations}

5FU: 5-fluorouracil; BM: Bone marrow; EGF: Epidermal growth factor; FPKM: Fragment per kilobase of exon per million fragments mapped; H\&E: Harris hematoxylin and eosin; HSPC: Hematopoietic stem and progenitor cell: FGFR: fibroblast growth factor receptor; LSK cells: Lineage negative, Sca-1 positive, cKit positive cells; LT-HSC: Long-term hematopoietic stem cells: HSC; OB: Osteoblast

\section{Acknowledgements}

Not applicable.

\section{Funding}

This research was supported by grants from the National Research Foundation (NRF) of Korea, which was funded by the Ministry of Education, Science and Technology (20110019355) and by the Bio \& Medical Technology Development Program of the Ministry of Science, ICT \& Future Planning (2012M3A9C6049716, 2014M3A9D5A01073598 and 2016M3A9B6026771).

\section{Availability of data and materials \\ Not applicable.}

\section{Authors' contributions}

Conception and design: KAY, JYC. Development of methodology: KAY, YJC, YSS, JHK, JYC. Acquisition of data: KAY, YJC, YSS, JHK. Analysis and interpretation of data: KAY, JYC. Writing, review, and/or revision of the manuscript: KAY, JYC. Administrative, technical, or material support: YSS, JYC. Study supervision: JYC. All authors read and approved the final manuscript.

\section{Competing interests}

The authors declare that they have no competing interests.

\section{Consent for publication}

Not applicable.

Ethics approval and consent to participate

Animal experiments were performed after approval from the IACUC of Seoul National University (approval \#: SNU-130916-2-1). 
Received: 13 December 2016 Accepted: 16 June 2017 Published online: 29 June 2017

\section{References}

1. Warr MR, Pietras EM, Passegue E. Mechanisms controlling hematopoietic stem cell functions during normal hematopoiesis and hematological malignancies. Wiley Interdiscip Rev Syst Biol Med. 2011;3:681-701.

2. Morrison SJ, Scadden DT. The bone marrow niche for haematopoietic stem cells. Nature. 2014;505:327-34.

3. Essers MA, Offner S, Blanco-Bose WE, Waibler Z, Kalinke U, Duchosal MA, et al. IFNalpha activates dormant haematopoietic stem cells in vivo. Nature. 2009:458:904-8

4. Wilson A, Laurenti E, Oser G, van der Wath RC, Blanco-Bose W, Jaworski M, et al. Hematopoietic stem cells reversibly switch from dormancy to selfrenewal during homeostasis and repair. Cell. 2008;135:1118-29.

5. Lucas D, Scheiermann C, Chow A, Kunisaki Y, Bruns I, Barrick C, et al. Chemotherapy-induced bone marrow nerve injury impairs hematopoietic regeneration. Nat Med. 2013;19:695-703.

6. Cao X, Wu X, Frassica D, Yu B, Pang L, Xian L, et al. Irradiation induces bone injury by damaging bone marrow microenvironment for stem cells. Proc Natl Acad Sci U S A. 2011;108:1609-14.

7. Lerner C, Harrison DE. 5-fluorouracil spares hemopoietic stem cells responsible for long-term repopulation. Exp Hematol. 1990;18:114-8.

8. Longley DB, Harkin DP, Johnston PG. 5-fluorouracil: mechanisms of action and clinical strategies. Nat Rev Cancer. 2003;3:330-8.

9. Zhao M, Ross JT, Itkin T, Perry JM, Venkatraman A, Haug JS, et al. FGF signaling facilitates postinjury recovery of mouse hematopoietic system. Blood. 2012;120:1831-42.

10. Eswarakumar VP, Lax I, Schlessinger J. Cellular signaling by fibroblast growth factor receptors. Cytokine Growth Factor Rev. 2005;16:139-49.

11. Johnson DE, Williams LT. Structural and functional diversity in the FGF receptor multigene family. Adv Cancer Res. 1993:60:1-41.

12. Miller DL, Ortega S, Bashayan O, Basch R, Basilico C. Compensation by fibroblast growth factor 1 (FGF1) does not account for the mild phenotypic defects observed in FGF2 null mice. Mol Cell Biol. 2000;20:2260-8.

13. Itkin T, Ludin A, Gradus B, Gur-Cohen S, Kalinkovich A, Schajnovitz A, et al. FGF-2 expands murine hematopoietic stem and progenitor cells via proliferation of stromal cells, c-kit activation, and CXCL12 down-regulation. Blood. 2012;120:1843-55.

14. Yeoh JS, van Os R, Weersing E, Ausema A, Dontje B, Vellenga E, et al. Fibroblast growth factor-1 and -2 preserve long-term repopulating ability of hematopoietic stem cells in serum-free cultures. Stem Cells. 2006;24:1564-72.

15. Caselli A, Olson TS, Otsuru S, Chen X, Hofmann TJ, Nah HD, et al. IGF-1mediated osteoblastic niche expansion enhances long-term hematopoietic stem cell engraftment after murine bone marrow transplantation. Stem Cells. 2013;31:2193-204.

16. Dominici M, Rasini V, Bussolari R, Chen X, Hofmann TJ, Spano C, et al. Restoration and reversible expansion of the osteoblastic hematopoietic stem cell niche after marrow radioablation. Blood. 2009;114:2333-43.

17. Olson TS, Caselli A, Otsuru S, Hofmann TJ, Williams R, Paolucci P, et al. Megakaryocytes promote murine osteoblastic HSC niche expansion and stem cell engraftment after radioablative conditioning. Blood. 2013;121:5238-49.

18. Du X, Xie Y, Xian CJ, Chen L. Role of FGFs/FGFRs in skeletal development and bone regeneration. J Cell Physiol. 2012;227:3731-43.

19. Greenbaum A, Hsu YM, Day RB, Schuettpelz LG, Christopher MJ, Borgerding $J N$, et al. CXCL12 in early mesenchymal progenitors is required for haematopoietic stem-cell maintenance. Nature. 2013:495:227-30.

20. Chan CK, Chen CC, Luppen CA, Kim JB, DeBoer AT, Wei K, et al. Endochondral ossification is required for haematopoietic stem-cell niche formation. Nature. 2009:457:490-4.

21. Yoon KA, Cho HS, Shin HI, Cho JY. Differential regulation of CXCL5 by FGF2 in osteoblastic and endothelial niche cells supports hematopoietic stem cell migration. Stem Cells Dev. 2012;21:3391-402.

22. Lee JH, Jeon SA, Kim BG, Takeda M, Cho JJ, Kim DI, et al. Nedd4 deficiency in vascular smooth muscle promotes vascular calcification by stabilizing pSmad1. J Bone Miner Res. 2017;32:927-38.

23. Zhang J, Niu C, Ye L, Huang H, He X, Tong WG, et al. Identification of the haematopoietic stem cell niche and control of the niche size. Nature. 2003:425:836-41.
24. Heissig B, Hattori K, Dias S, Friedrich M, Ferris B, Hackett NR, et al. Recruitment of stem and progenitor cells from the bone marrow niche requires MMP-9 mediated release of kit-ligand. Cell. 2002;109:625-37.

25. Bord S, Frith E, Ireland DC, Scott MA, Craig Jl, Compston JE. Megakaryocytes modulate osteoblast synthesis of type-I collagen, osteoprotegerin, and RANKL. Bone. 2005:36:812-9.

26. Kacena MA, Shivdasani RA, Wilson K, Xi Y, Troiano N, Nazarian A, et al. Megakaryocyte-osteoblast interaction revealed in mice deficient in transcription factors GATA-1 and NF-E2. J Bone Miner Res. 2004;19:652-60.

27. Lemieux JM, Horowitz MC, Kacena MA. Involvement of integrins alpha(3)beta(1) and alpha(5)beta(1) and glycoprotein Ilb in megakaryocyteinduced osteoblast proliferation. J Cell Biochem. 2010;109:927-32.

28. Zhou BO, Yue R, Murphy MM, Peyer JG, Morrison SJ. Leptin-receptorexpressing mesenchymal stromal cells represent the main source of bone formed by adult bone marrow. Cell Stem Cell. 2014;15:154-68.

29. Mizoguchi T, Pinho S, Ahmed J, Kunisaki Y, Hanoun M, Mendelson A, et al. Osterix marks distinct waves of primitive and definitive stromal progenitors during bone marrow development. Dev Cell. 2014;29:340-9.

30. Lee-Thedieck C, Rauch N, Fiammengo R, Klein G, Spatz JP. Impact of substrate elasticity on human hematopoietic stem and progenitor cell adhesion and motility. J Cell Sci. 2012;125:3765-75.

31. Mendelson A, Frenette PS. Hematopoietic stem cell niche maintenance during homeostasis and regeneration. Nat Med. 2014;20:833-46.

32. Doan PL, Himburg HA, Helms K, Russell JL, Fixsen E, Quarmyne M, et al. Epidermal growth factor regulates hematopoietic regeneration after radiation injury. Nat Med. 2013:19:295-304.

33. Passegue E, Wagers AJ, Giuriato S, Anderson WC, Weissman IL. Global analysis of proliferation and cell cycle gene expression in the regulation of hematopoietic stem and progenitor cell fates. J Exp Med. 2005;202:1599-611.

34. Xiao L, Liu P, Li X, Doetschman T, Coffin JD, Drissi H, et al. Exported 18-kDa isoform of fibroblast growth factor-2 is a critical determinant of bone mass in mice. J Biol Chem. 2009;284:3170-82

35. Montero A, Okada Y, Tomita M, Ito M, Tsurukami H, Nakamura T, et al. Disruption of the fibroblast growth factor-2 gene results in decreased bone mass and bone formation. J Clin Invest. 2000;105:1085-93.

36. Nakamura Y, Arai F, Iwasaki H, Hosokawa K, Kobayashi I, Gomei Y, et al. Isolation and characterization of endosteal niche cell populations that regulate hematopoietic stem cells. Blood. 2010;116:1422-32.

37. Calvi LM, Adams GB, Weibrecht KW, Weber JM, Olson DP, Knight MC, et al. Osteoblastic cells regulate the haematopoietic stem cell niche. Nature. 2003:425:841-6.

38. Karanu FN, Murdoch B, Gallacher L, Wu DM, Koremoto M, Sakano S, et al. The notch ligand jagged-1 represents a novel growth factor of human hematopoietic stem cells. J Exp Med. 2000;192:1365-72.

39. Marie PJ, Coffin JD, Hurley MM. FGF and FGFR signaling in chondrodysplasias and craniosynostosis. J Cell Biochem. 2005:96:888-96.

40. Schepers K, Hsiao EC, Garg T, Scott MJ, Passegue E. Activated Gs signaling in osteoblastic cells alters the hematopoietic stem cell niche in mice. Blood. 2012;120:3425-35

41. Calvi LM, Bromberg O, Rhee Y, Weber JM, Smith JN, Basil MJ, et al. Osteoblastic expansion induced by parathyroid hormone receptor signaling in murine osteocytes is not sufficient to increase hematopoietic stem cells. Blood. 2012;119:2489-99.

42. Ding L, Morrison SJ. Haematopoietic stem cells and early lymphoid progenitors occupy distinct bone marrow niches. Nature. 2013;495:231-5.

43. Weber JM, Forsythe SR, Christianson CA, Frisch BJ, Gigliotti BJ, Jordan CT, et al. Parathyroid hormone stimulates expression of the notch ligand Jagged1 in osteoblastic cells. Bone. 2006;39:485-93.

44. Zhou X, Zhang Z, Feng JQ, Dusevich VM, Sinha K, Zhang H, et al. Multiple functions of Osterix are required for bone growth and homeostasis in postnatal mice. Proc Natl Acad Sci U S A. 2010;107:12919-24. 